\title{
EDITORIAL
}

\section{The key or just a cog in the wheel to operability assessment?}

\author{
Nick H. Kim
}

$\mathbf{T}$ he ability to measure the hydrostatic pressure in a capillary bed $\left(P_{\mathrm{c}}\right)$ was an impetus for the development of the arterial occlusion method [1]. The relevance of $P_{\mathrm{c}}$ in pulmonary circulation is its role in pulmonary oedema formation. In order to accurately measure $P_{c}$, the isogravimetric method and the double occlusion technique have served as standards [2, 3]. However, both techniques require surgical isolation of the organ. The pulmonary artery occlusion technique was developed for in vivo use with a standard fluid-filled pulmonary artery catheter. When incorporated, the technique enabled $P_{c}$ measurements as part of a standard right heart catheterisation procedure. But interest in this technique appeared to wane after discovering that the pulmonary artery occlusion technique, rather than measuring $P_{\mathrm{c}}$, was measuring a pre-capillary pressure in the $>80-100 \mu \mathrm{m}$ calibre range $[1,4]$. Although this limited its application in pulmonary oedema research, the technique would later find renewed interest in the field of pulmonary hypertension $(\mathrm{PH})[4,5]$.

Of the PH subtypes, the occlusion technique seemed especially applicable for chronic thromboembolic pulmonary hypertension (CTEPH). In addition to the chronic material in the proximal pulmonary arteries, which respond to removal by pulmonary endarterectomy (PEA), these patients also have varying degrees of distal, small vessel arteriopathy which is beyond the reach of endarterectomy [6]. Although the presence of concomitant small vessel arteriopathy does not preclude successful PEA, it accounts for post-operative residual PH and contributes to the leading cause of morbidity and mortality following endarterectomy [7, 8]. Despite advances in imaging technology and pre-operative evaluations prior to PEA, the degree of small vessel disease in CTEPH cannot be measured or quantified. This inability to accurately assess the component of small vessel arteriopathy remains a key reason why CTEPH operability assessment remains subjective when predicting post-operative haemodynamic outcome. Accordingly, CTEPH operability assessment relies heavily on experience and opinion, and is variable from centre to centre and even from patient to patient. Although the occlusion technique may fall short in estimating $P_{\mathrm{c}}$, by analysing the status of the small

Division of Pulmonary and Critical Care Medicine, University of California San Diego, La Jolla, CA, USA.

CORRESPONDENCE: N.H. Kim, Division of Pulmonary and Critical Care Medicine, University of California San Diego, 9300 Campus Point Drive, MC 7381, La Jolla, CA 92037, USA. E-mail: h33kim@ucsd.edu arterial vessels, this catheter-based technique may have a role in objectifying this aspect of CTEPH operability.

In the current issue of the European Respiratory Journal, TOSHNER et al. [9] have reported the largest series to date applying the pulmonary artery occlusion technique in CTEPH. They report a correlation between pre-operative upstream resistance (Rup) measured with the occlusion technique and PEA clinical operability assessment. They also analysed and reported Rup in post-PEA patients and a cohort of PAH patients as comparator groups. This report follows our earlier work correlating Rup with post-operative outcome in CTEPH [10]. There are some similarities and differences between the two reports which the authors nicely address. One important difference, also addressed in the manuscript, has to do with the clinical assessment of operability, a topic of ongoing debate both in practice as well as among CTEPH/PEA experts.

For an operable disease, we lack a pre-operative classification system in CTEPH. Due to the complex nature of PEA, surgeonand centre-experience matter and correlate with outcome [11]. High volume PEA centres may be more inclined to offer surgery to CTEPH patients who may otherwise be turned down by less experienced centres. Those CTEPH patients turned down for PEA are often labelled inoperable and find themselves receiving trial medical therapy [12]. Although there may be other contributing factors to bypass potentially curative surgery, this process is not helped by the absence of clear operability guidelines. Even though the current investigators are some of the leading experts in the field of CTEPH and PEA, the preoperative judgement of inoperability remains subjective rather than absolute. In contrast, patients with CTEPH who received PEA from an experienced surgeon and still have significant $\mathrm{PH}$ or poor outcome may be more fitting of the inoperable classification. For now, an argument could be made that CTEPH patients are better categorised as either being operated or not-operated. Until we have a better and more objective way of discerning operability in $\mathrm{CTEPH}$, this will be an ongoing point of discussion.

Whether the pulmonary artery occlusion technique could be the key to operability in CTEPH remains unclear. But the authors have taken a major stride forward with this report and have led us to important questions and guidance towards future investigations. The relatively high Rup in all cohorts, including the PAH patients, raises the question as to whether we have found the right sweet spot with this version of the occlusion method. Will another occlusion analysis method separate the $\mathrm{PAH}$ and CTEPH patients with significant concomitant small vessel disease from patients with primarily proximal disease 
amenable to PEA [13]? Should multiple occlusion samplings at different locations be factored to derive an aggregate Rup? Or instead, should location for these samplings be guided by a lung perfusion scan analysing intact perfusion beds? Could studying operated patients with residual $\mathrm{PH}$ before and after medical therapy targeting the small vessels provide treatment data or guidance? In spite of all these and other questions, the occlusion technique is in a unique position to offer more objective assessment in an otherwise subjective discipline of operability determination.

\section{STATEMENT OF INTEREST}

None declared.

\section{REFERENCES}

1 Hakim TS, Kelly S. Occlusion pressures vs. micropipette pressures in the pulmonary circulation. J Appl Physiol 1989; 67: 1277-1285.

2 Parker JC, Kvietys PR, Ryan KP, et al. Comparison of isogravimetric and venous occlusion capillary pressures in isolated dog lungs. J Appl Physiol 1983; 55: 964-968.

3 Townsley MI, Korthuis RJ, Rippe B, et al. Validation of double vascular occlusion method for $\mathrm{Pc}_{\mathrm{C}, \mathrm{I}}$ in lung and skeletal muscle. J Appl Physiol 1986; 61: 127-132.

4 Kafi SA, Melot C, Vachiery JL, et al. Partitioning of pulmonary vascular resistance in primary pulmonary hypertension. J Am Coll Cardiol 1998; 31: 1372-1376.
5 Fesler P, Pagnamenta A, Vachiéry J-L, et al. Single arterial occlusion to locate resistance in patients with pulmonary hypertension. Eur Respir J 2003; 21: 31-36.

6 Moser KM, Bloor CM. Pulmonary vascular lesions occurring in patients with chronic major vessel thromboembolic pulmonary hypertension. Chest 1993; 103: 685-692.

7 Jamieson SW, Kapelanski DP, Sakakibara N, et al. Pulmonary endarterectomy: experience and lessons learned in 1,500 cases. Ann Thorac Surg 2003; 76: 1457-1462.

8 Archibald CJ, Auger WR, Fedullo PF, et al. Long-term outcome after pulmonary thromboendarterectomy. Am J Respir Crit Care Med 1999; 160: 523-528.

9 Toshner M, Suntharalingam J, Fesler P, et al. Occlusion pressure analysis role in partitioning of pulmonary vascular resistance in CTEPH. Eur Respir J 2012; 40: 612-617.

10 Kim NH, Fesler P, Channick RN, et al. Preoperative partitioning of pulmonary vascular resistance correlates with early outcome after thromboendarterectomy for chronic thromboembolic pulmonary hypertension. Circulation 2004; 109: 18-22.

11 Mayer E, Jenkins D, Lindner J, et al. Surgical management and outcome of patients with chronic thromboembolic pulmonary hypertension: results from an international prospective registry. J Thorac Cardiovasc Surg 2011; 141: 702-710.

12 Pepke-Zaba J, Delcroix M, Lang I, et al. Chronic thromboembolic pulmonary hypertension (CTEPH): results from an international prospective registry. Circulation 2011; 124: 1973-1981.

13 Pellett AA, Johnson RW, Morrison GG, et al. A comparison of pulmonary arterial occlusion algorithms for estimation of pulmonary capillary pressure. Am J Respir Crit Care Med 1999; 160: 162-168. 